\title{
IMPACT OF COLOR MATCHING PRIMARIES ON OBSERVER MATCHING: PART II - OBSERVER VARIABILITY
}

\author{
Jiaye $\mathrm{Li}^{\mathrm{a} *}$, Peter Hanselaer ${ }^{\mathrm{a}}$, Kevin A. G. Smet ${ }^{\mathrm{a}}$ \\ ${ }^{a}$ KU Leuven, ESAT/Light\&Lighting Laboratory, Ghent, BELGIUM
}

*jiaye.li@kuleuven.be, *https://orcid.org/0000-0001-8900-3264

ORCiD Kevin Smet: https://orcid.org/0000-0003-3825-6274

\begin{abstract}
In this study, the observer variability in a $10^{\circ}$ achromatic color matching experiment using systematically different primaries under immersive, perceptually neutral (4673 K) and stable adapted viewing conditions has been investigated for 4 different sets of CIE color matching functions (CMFs). The matching reference was illuminated by a spectrally broadband light source at $70 \mathrm{~cd} / \mathrm{m}^{2}$ and $4613 \mathrm{~K}$. Fifty-four observers varying in age, gender, ethnicity and eye color participated. The results indicate that certain wavelength regions are more sensitive to generate inter-observer variability, such as when a short wavelength primary $(404 \mathrm{~nm})$ was applied. Furthermore, of the primary sets investigated, that with peak-wavelengths (636-521-447 nm) close to the 'typical' RGBprimaries widely known and used in industry had the smallest inter-observer variability. In addition to the significant effect of primary set and CMF set, a significant effect of age on matching variability is also found, whereby the change of the blue primary resulted in a substantial difference in dispersion between younger and older observers, likely caused by age-induced yellowing of the lens. Furthermore, different primary sets tend to have a different impact on intra-observer variability than on inter-observer variability. Finally, by comparing the observer variability in the current work with previous studies, the CIE standard deviate observer is found to substantially underestimate the inter-observer variability, especially for the primary sets containing a $404 \mathrm{~nm}$ LED. Our observers also tend to have similar to smaller inter-variability than those reported in literature, possibly due to methodological differences.
\end{abstract}

Keywords: Color Matching, Color Matching Functions, Matching primaries, Observer variability

\section{Introduction}

A fundamental property of normal human color vision is trichromacy, i.e. that a color match can be found for any light (visual stimulus) by varying the optical power of three primaries. Trichromacy can be characterized by three spectral response functions: color matching functions (CMFs). There are substantial variations in the physiology of individual observers, resulting in different individual CMFs. Therefore, the International Commission for Illumination (CIE) standardized the specification of color by defining CMFs of an average or a standard observer for practical purposes.

By now, several CIE CMF sets for $2^{\circ}$ or $10^{\circ}$ field of view (FOV) have been defined: CIE $19312^{\circ}$ (CIE 2004), CIE $196410^{\circ}$ (CIE 2004) and the CIEPO06 $2^{\circ}$ and $10^{\circ}$ standard 
colorimetric observers (CIE 2006). An overview of some details of the chosen CMF sets is given in Table 1.

Table 1: Overview of some details of the chosen CMF sets

\begin{tabular}{|c|c|c|c|c|c|}
\hline CMF set & Field of view & Year & $\begin{array}{l}\text { Color matching } \\
\text { dataset(s) }\end{array}$ & $\begin{array}{l}\text { Number of } \\
\text { observers }\end{array}$ & Reference \\
\hline CIE 1931 & $2^{\circ}$ & 1931 & Guild + Wright & $7+10$ & $\begin{array}{l}\text { (Wright 1929; Guild } \\
\text { 1931) }\end{array}$ \\
\hline CIE 1964 & $10^{\circ}$ & 1964 & $\begin{array}{l}\text { Stiles \& Burch }+ \\
\text { Speranskaya }\end{array}$ & $49+27$ & $\begin{array}{l}\text { (Stiles and Burch 1955a; } \\
\text { Speranskaya 1959; CIE } \\
\text { 1986) }\end{array}$ \\
\hline CIEPO06 $2^{\circ}$ & $2^{\circ}$ & $\begin{array}{l}2006 \\
(2015)\end{array}$ & Stiles \& Burch & 49 & $\begin{array}{l}\text { (Stockman and Sharpe } \\
\text { 2000; CIE 2006; CIE 2015) }\end{array}$ \\
\hline CIEPO06 $10^{\circ}$ & $10^{\circ}$ & $\begin{array}{l}2006 \\
(2015)\end{array}$ & Stiles \& Burch & 49 & $\begin{array}{l}\text { (Stockman and Sharpe } \\
\text { 2000; CIE 2006; CIE 2015) }\end{array}$ \\
\hline
\end{tabular}

The CIE $19312^{\circ}$ standard observer is currently the most widely used in industry and technology (CIE 2004). This CMF set was derived based on color matching experiments of Guild (Guild 1931) and Wright (Wright 1929), whereby observers tried to match the color of an arbitrary photopic reference stimulus (test field) by adjusting a match field composed of an additive mixture of three monochromatic primaries.

In 1959, Stiles and Burch published the largest set of centrally-viewed $10^{\circ} \mathrm{CMFs}$ composed of data for 49 observers (Stiles and Burch 1955a). The color matching data collected by Stiles and Burch were used to form the basis for CIE 1964 CMFs (CIE 1986), as well as to develop the CIE Standard Deviate Observer (CIE 1989).

As experimentally derived CMFs can be converted by a linear transform to cone fundamentals (CFs) representative of (but not identical to) the $L, M, S$ cone spectral sensitivities (absorptance), the Stiles data were also used as the basis for the cone fundamentals proposed by Stockman and Sharpe (Stockman and Sharpe 2000) and for the physiologically relevant CIE0P06 CMFs proposed in 2006 (CIE 2006). In addition to a $2^{\circ}$ and $10^{\circ} \mathrm{CMF}$ set, CIEPO06 also includes a parametric model that allows to calculate CMFs for different observer ages and stimulus field-of-views (FOV) by accounting for their effect on physiological factors, such as the absorption by the macular pigment, lens and other ocular media, as well as the peak densities of the photopigments (CIE 2006).

The CIE physiological observer CIEPO06 model (with FOV and observer age as only parameters) was extended by (Asano, Fairchild, and Blondé 2016) to simulate the CMFs of individual observers by adding eight additional parameters: deviations from averages for lens pigment density, macular pigment density and optical density and peak wavelength shifts of the L, M, S cone photopigments, respectively. Based on an extensive literature study, Asano identified the variability of each of the physiological parameters to enable the simulation of color-normal individual CMFs. An advantage of the Individual Colorimetric Observer model of Asano is that it allows to estimate the expected observer variability in the XYZ tristimulus values, and hence in predicted visual matches; for example, by calculating the XYZ covariance matrix as proposed by Nimeroff (Nimeroff et al. 1961) or, for example, to investigate the effect 
of observer variability on various color measures, such as color rendition fidelity (Murdoch and Fairchild 2019).

Color matching is generally characterized by a large inter-observer variability and the concept of an average observer has its limitations. There is no guarantee that for a particular observer a predicted match would actually result in a visual match.

During the past a few decades, researchers attempted to evaluate the observer metamerism, many using the trichromatic matching data, or a subset thereof, collected in Stiles and Burch's experiment (Stiles and Burch 1959). In 1970, Allen (Allen 1970) attempted to quantify observer metamerism by statistically analyzing the variances and covariances of 20 CMFs selected from the Stiles and Burch data, based on their color matching experience (Wyszecki and Stiles 1982). In 1985, Nayatani and Takahama et al. (Nayatani et al. 1985; Takahama et al. 1985), using the same 20 sets of CMFs, successfully derived four deviation functions, which can be used to evaluate the degree of observer metamerism and from which confidence ellipsoids of matching chromaticities can be constructed. This method was used for defining the CIE standard Deviate Observer and the metamerism index, published by CIE in 1989 (CIE 1989). The CIE standard Deviate Observer model (CIE 1989) aimed to evaluate observer variability by predicting the degree of color difference found by standard deviate observers between a pair of metameric colors. However, many researchers reported (North and Fairchild 1993; Rich and Jalijali 1995; Alfvin and Fairchild 1997; Sarkar, L. Blondé, et al. 2010) that the CIE standard deviate observer model tends to under-evaluate the matching variability among real observers. Possible reasons could be the exclusion of more than half of the full set of Stiles-Burch's observers in its derivation and an improper normalization of the original color matching data (North and Fairchild 1993).

Articles by Thornton (Thornton 1992), North and Fairchild (North and Fairchild 1993), and Shaw and Fairchild (Shaw and Fairchild 2002) have reported experimental results that show large amounts of inter-observer variability and, in some cases, significant differences from the matches predicted using the CIE 1964 standard observer functions. Thornton (Thornton 1997) found that the inter-observer differences appeared to be large for anti-prime color (AP: 497-579-653 nm) monochromatic matching primaries, but small for prime color (PC: 452-533-607 nm) spectral composition. Since the average age of Thornton's six observers was 56 years and the average age of the CIE observer was 32 years, lens yellowing could be a factor explaining some of the discrepancies between the average data of the two groups (Shapiro et al. 1994). In a re-analysis of Thornton's data, Abraham (Ábrahám et al. 1995) did indeed find that there was a difference between the mean chromaticity of matches made by old and young observers when using the AP primaries, causing increased overall variability. However, there was none when using the PC primaries. Abraham also found that the magnitude of dispersions of the matching chromaticities were consistent with lens transmission, but not the direction (Ábrahám et al. 1995).

Even with the use of the CIEPO06 model, which theoretically should be able to account for observer age and field size, the use of an observer's actual age does not necessarily result in CMFs that are representative for that particular observer, due to unsystematic observer variability and/or because one or more age-independent physiological factors have not been included (Sarkar, Blondé, et al. 2009). The usefulness of the CIEPO06 model, in its current form, to predict the average observer for a given age group may, therefore, be limited (Sarkar 
et al. 2011). Shi et al. (Shi et al. 2017) indicated that observers have a substantially different perception, even within the same age group and for the same visual field. In addition, Sarkar (Sarkar 2011a) has shown that the Stiles \& Burch (Stiles and Burch 1959) color matching data can be subdivided into 8 observer categories and that the CIE $196410^{\circ}$ standard observer, which is very similar to the CIEPO06 $10^{\circ}$ observer, corresponds to only $8 \%$ of the observers. Importantly and unfortunately, no category accounted for more than $25 \%$ of the observers. Asano (Asano and Fairchild 2020) recently derived categorical observers from their individual colorimetric observer model and found that for general color imaging applications with not too narrowband primaries, up to 10 categorical observers can represent the color-normal population, while more (up to 50) are required, for example, for laser-line primaries.

The extension of the CIEPO06 model to include additional parameters affecting individual observer CMFs in the Asano model has been shown to result in improved predictions of real observer metamerism (Asano, Fairchild, and Blondé 2016). Although an accurate assessment of observer metamerism may have been less critical in the past when stimuli had rather spectrally broadband characteristics, it is not so now with the development and advent of the increasingly narrowband spectra in wide-gamut, light-emitting-diode (LED), quantum dot and laser based displays and lighting (Fairchild and Wyble 2007; Sarkar, L. Blondé, et al. 2010; Sarkar 2011b; Long and Fairchild 2014; Asano 2015).

Some other possible (physiological) mechanisms and factors could also lead to a loss of generality of the color matching functions and to a large dispersion or variability of the matches. In recent studies, gender has also been found to have an impact on color discrimination (Haddad et al. 2009; Jain et al. 2010). It was found that females can see more color shades and have better color discrimination than males. It would, therefore, be interesting to consider the impact of gender when studying color matching variability. Furthermore, cultural differences could also lead to different matching results. Rich et al. (Rich and Jalijali 1995) reported that there was a higher degree of consistency and reliability in the Japanese observations, possibly due to the genetic pool of Asian observers being more uniform than that of Caucasians. In addition, eye color, which is determined by both the pigmentation of the eye's iris, and the wavelength dependence of the scattering of light by the turbid medium in the stroma of the iris (Prota et al. 1998; Wielgus and Sarna 2005) could have an impact on color matching results as it would add a veil of colored light transmitted through the iris.

In the current work, an achromatic matching experiment was performed using multiple LED primaries by 54 observers varying in age, gender, ethnicity and eye color. The peak wavelengths of the primaries were chosen based on some of the findings reported earlier by Thornton (Thornton 1992), Sarkar (Sarkar 2011b) and Asano (Asano 2015). Each primary set includes one reddish, one greenish and one bluish light. Maxwellian matches were made using a $10^{\circ}$ FOV bipartite color matching setup, in which observers can adjust the optical power of the LED primaries in the matching field to match the color appearance of a neutral broadband reference field. The luminance of both the matching field and reference field remained fixed at $70 \mathrm{~cd} / \mathrm{m}^{2}$. The bipartite field was embedded in a $70 \mathrm{~cd} / \mathrm{m}^{2}$ background to keep the observer's state of adaptation constant throughout the experiment. In the analysis, the impact of the choice of CMFs and primaries on observer variability is investigated by calculating the collected data with the CIE $19312^{\circ}, 196410^{\circ}, 20062^{\circ}$ and $200610^{\circ} \mathrm{CMF}$ sets for different primary sets. Although using $2^{\circ} \mathrm{CMFs}$ is physiologically not appropriate for matches made in a $10^{\circ}$ field of 
view - because of the differences in densities of the macular and cone pigments, as well as other contributing factors such as chromatic aberration, area summation, etc. - we included both $2^{\circ}$ and $10^{\circ} \mathrm{CMFs}$ in order to illustrate the potential impact of these differences on the matching results. The possible effects of some physiological factors, such as observer age, gender, ethnicity and eye color, on inter-observer variability are also investigated. The intraobserver variability is compared to inter-observer variability grouped per primary-set. In addition, the matching variability in the current study is compared to that predicted by the CIE standard deviate observer and that found in Asano's work.

\section{Methods}

\subsection{Experimental setup}

The same experimental setup (see Fig. 1) was used as in (Li et al. 2020). The specially designed viewing booth ( $\mathrm{Li}$ et al. 2019a; Li et al. 2019b) allows observers to make Maxwellian color matches in reflective / object appearance mode under visually immersive conditions.

(a)

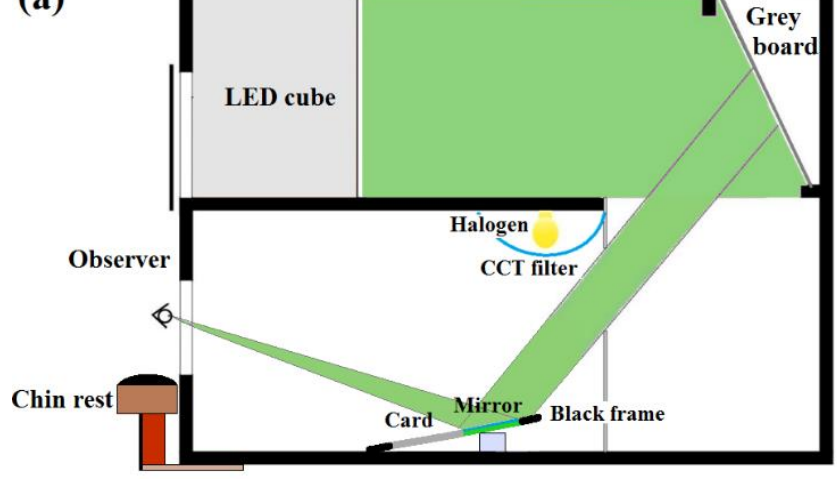

(b)

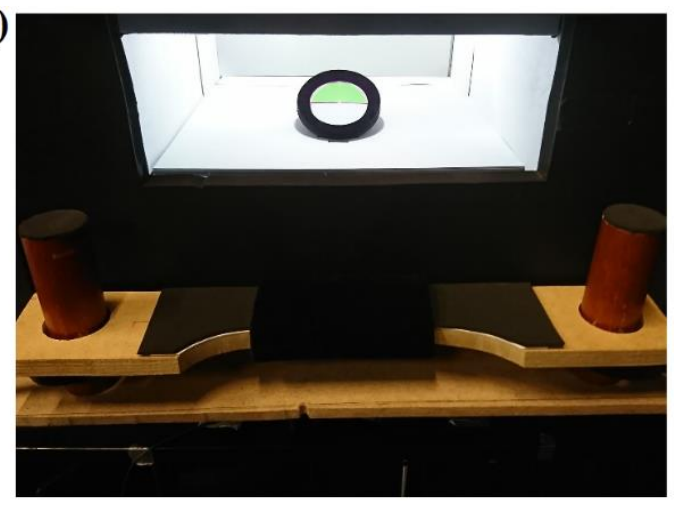

Fig. 1. Experimental Setup: (a) Schematic of the viewing booth; (b) The $10^{\circ} \mathrm{FOV}$ bipartite field composed of a mirror (reflecting the image of a grey board illuminated by LEDs) and the reference card (illuminated by a filtered halogen). The bipartite field was specially designed with the shape tuned to be perceptually circular from the observer's viewing position. A chin rest was used to fix the observer's position.

In the viewing booth, a $10^{\circ}$ bipartite circular field was presented to observers, one halffield for matching and another for the reference (see Fig. 1b).

A calibrated, tunable 15-channel LED source (Thouslite) was used to generate a narrowband matching stimulus. The match field was composed of a slightly tilted half-circular mirror that reflected the image of a gray diffusive board uniformly illuminated by three selected primaries (channels) of the LED cube. The diffusive board and LEDs were both located in the integrating chamber (top half of the viewing booth), which was covered by highly reflective $(\approx 87.8 \%)$ and non-fluorescent paper to minimize spatial non-uniformity of the reflected light. This mirror setup of the matching field allowed to provide an indistinguishable stimulus (see Fig. 1b), with the same lightness and color as an opaque, gray (spectrally neutral) card illuminated by a filtered broadband halogen source $\left(L=70 \mathrm{~cd} / \mathrm{m}^{2}, C C T=4613 \mathrm{~K}\right.$, $D u v=0.0056$, calculated with CIE $19312^{\circ} \mathrm{CMFs}$ ) presented in the reference field (Flecy and Smet 2015; Li et al. 2019a; Li et al. 2019b); although the color changes in the matching field 
might break this illusion. Both the tilted angle and shape of the match and reference field were specially designed such that the bipartite field appeared approximately circular and with a $10^{\circ}$ FOV at a viewing distance of $0.45 \mathrm{~m}$. A $2^{\circ}$ black frame has been added to the $10^{\circ}$ bipartite field in order to minimize the potential effects of simultaneous contrast and induction (Blackwell and Buchsbaum 1988).

The bipartite field was presented in an immersive $\left(>150^{\circ}\right)$, uniform grey surround to provide observers with a fixed adaptation state (Ma et al. 2018). The luminance of both the bipartite field and the surround was set at as high as possible $\left(70 \pm 2 \mathrm{~cd} / \mathrm{m}^{2}\right)$, given the physical limitations of the setup, to suppress rod intrusion. Matches were made under immersive and steady-state adaptive conditions, instead of the typical stimulus presentation in aperture mode under dark-adapted viewing conditions as used in most other studies (Sarkar 2011b; Asano 2015). However, such conditions are less representative of daily viewing conditions, while dark-adapted conditions also tend to lead to a higher observer variability (Smet et al. 2014) and a different color perception (Judd 1961; Braun et al. 1996; Katoh et al. 1998; Henley and Fairchild 2000; Cuttle 2008; Kuriki 2015).

The viewing booth was calibrated, which allowed observers to adjust the chromaticity of the matching stimulus in the CIE $1976 u$ 'v' diagram using a joystick (Logitech Extreme 3D Pro) connected to a control laptop. After the observer had finished making all matches, a calibrated spectroradiometer (Ocean Optics QE65 Pro) was used to measure their spectral radiance. Prior to the experiment, a series of measurements were done to check the stability of the channels of the Thouslite LEDcube. During a 5-hour period, the differences in chromaticity and relative luminance were respectively below 0.001 ( $u$ ' $v$ ' unit) and $0.02 \%$ for all the channels after a 15 minutes' warm-up period. When the matching field was set to the same chromaticity and luminance of the reference field, its chromaticity and relative luminance changed with less than 0.0007 ( $u$ 'v' units) and $0.001 \mathrm{~cd} / \mathrm{m}^{2}$ (measurement accuracy) over 90 minutes (the maximum time for an observer to finish the experiment session).

\subsection{LED primaries}

The Thouslite LED cube contains 15 channels, from which eight were chosen as matching primaries. These primaries were selected based on Asano (Asano 2015), Sarkar (Sarkar 2011d) and Thornton's work (Thornton 1992); more details can be found in PART I (Li et al. 2020). Eight primary combinations (referred to as 'primary set') were generated, each containing one 'red', one 'green' and one 'blue' primary: P1: 636-502-447 nm, P2: 636-502-404 nm, P3: 636521-447 nm, P4: 636-521-404 nm, P5: 598-521-447 nm, P6: 598-521-404 nm, P7: 673-521447 nm, P8: 636-521-475 nm.

\subsection{Experimental procedure}

At the start of the experiment, observers were given brief verbal instructions on the experiment procedure. During this time (approx. 5 minutes), they were allowed to adapt to the viewing (booth) conditions. Observers were seated in front of the viewing booth, with their head in a fixed position using a chin rest. The chin rest was adjusted to ensure observers only saw the reflection of the diffusive board in the match field mirror. Before starting the actual experiment, 
observers were asked to make one trial match to get familiar with the matching procedure. Observers were instructed to ignore any non-uniformity in the color of the central region (due to Maxwell's spot) of the bipartite field, as is customary in most color matching experiments (Houser and Hu 2004; Sarkar 2011b; Asano 2015). They had to make four matches for each primary set, starting from a different initial chromaticity. These were selected such that they were within the boundary of all the gamuts corresponding to the eight primary sets at $70 \mathrm{~cd} / \mathrm{m}^{2}$, while also being evenly distributed along the hue circle to avoid starting bias (Ma et al. 2019).

In each experiment, a total of 33 (8 primary sets $\mathrm{x} 4$ starting points +1 trial) color matches were made by each observer in a random order. After making a match, observers have to press a joystick button to save their setting and continue to the next one. During the experiment, observers could ask for a break whenever they felt fatigued, after which they had to re-adapt to the experimental background before continuing the matching. The average experiment time was about 1 hour, varying from 30 to 90 minutes for different observers.

\subsection{Observers}

Fifty-four color-normal observers ( 24 females, 30 males) participated in the experiments. Their color vision was tested with the Ishihara 24 plate test and the FM 100 hue test before the experiment. The average age of observers was $33 \pm 11$ years. Note that more detailed information of observers (age, gender, ethnicity, eye color) were already reported in PART I (Li et al. 2020). All the observers were instructed to take off their eyeglasses or contact lenses while doing the experiment, as some of them could contain UV filters that would affect the blue primary $(404 \mathrm{~nm})$.

\section{Results and discussion}

Overall, a total of 1728 color matches have been made. A mean visual match spectrum was calculated by averaging the spectra of the four matches made for each starting chromaticity. The obtained color matching results are reported and analyzed in the following subsections. Firstly, some general findings on the impact of primary set and CMF set on the inter-observer variability are discussed. Then, the impact of the within-subject (primary set, CMF set) and between-subject factors (observer age, gender, ethnicity and eye color) on the inter-observer variability, characterized by the covariance ellipses of the individual observer matches, was statistically analyzed using Box's M test (Box 1949). Secondly, the impact of the primary set and CMF set on the intra-observer variability were also characterized in terms of standard deviation (SD) ellipses. The inter- and intra-observer variability are compared for each primary set separately. Finally, the observer variability found in the current study was also compared to that predicted by the CIE standard deviate observer (CIE 1989), and that found in Asano's (Asano 2015; Asano, Fairchild, Blondé, et al. 2016) and Sarkar's work (Sarkar, Laurent Blondé, et al. 2010).

\subsection{Inter-observer variability}

The visual matches made during the experiment were predicted using the CIE $19312^{\circ}$ (CIE 
2018), CIE $196410^{\circ}$ (CIE 2018), and CIEPO06 $2^{\circ}$ and CIEPO06 10 (CIE 2006) standard observers, and converted to CIE $1976 u$ 'v'-like chromaticity using the same standard transform for all CMF sets. The tristimulus values of the reference color is also calculated. Note that the chromaticity differences between the spectrally broadband reference calculated using each of the $4 \mathrm{CMF}$ sets were so small $\left(D E u^{\prime} v^{\prime}=0.0010 \pm 0.0006\right)$ that the use of the same XYZ-to-u'v' transform for all CMF sets is warranted, even though the transform equations were developed for CIE $19312^{\circ} \mathrm{CMFs}$.

The inter-observer SD-ellipses, centered at the origin to better illustrate differences in variability (Friendly and Sigal 2018), are plotted in $u$ 'v' coordinates in Fig. 2 (calculated using $4 \mathrm{CMF}$ sets and grouping per primary set).
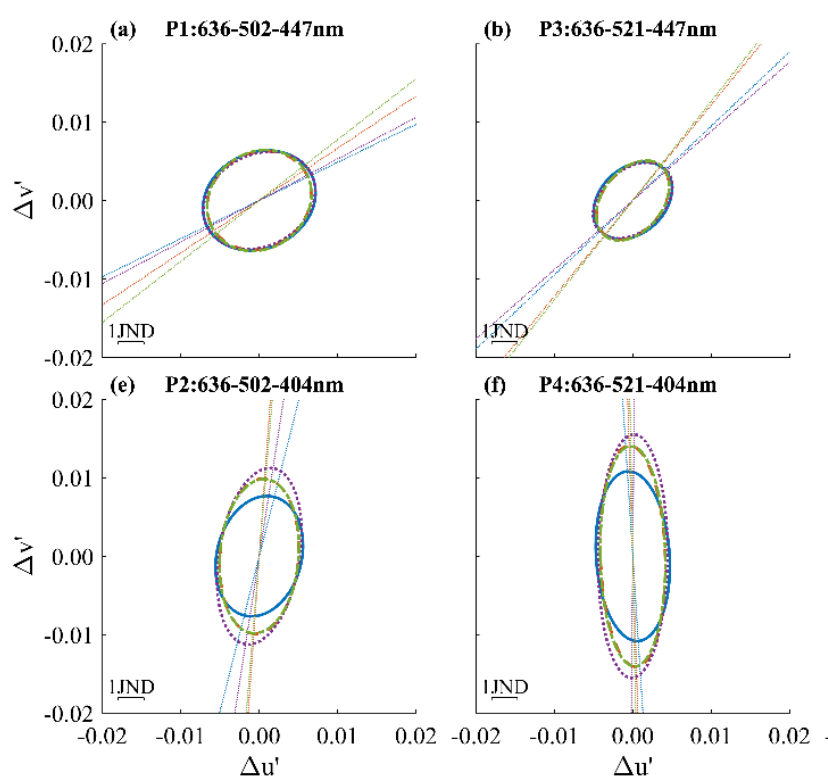

SD ellipse(CIE $\left.19312^{\circ}\right)$ : inter-variability (b) $\quad P 3: 636-521-447 \mathrm{~nm}$

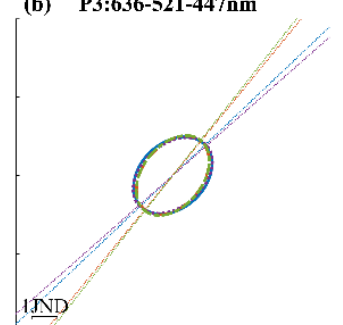

(f) P4:636-521-404nm SD ellipse $\left(\right.$ CIEPO06 $\left.^{\circ}\right)$ : inter-variability

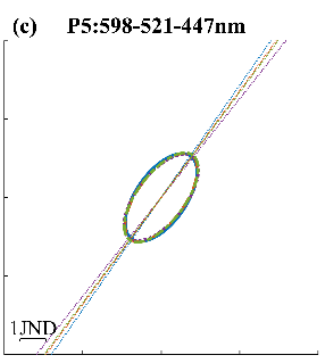

(g) P6:598-521-404nm

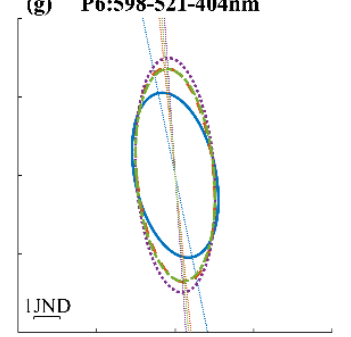

$\Delta \mathrm{u}^{\prime}$ (d) P7:673-521-447nm

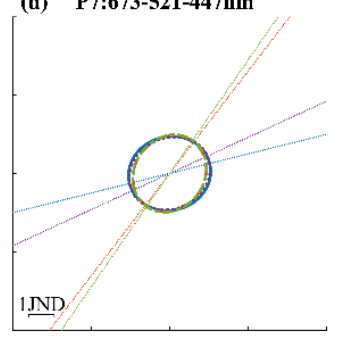

(h) P8:636-521-475nm

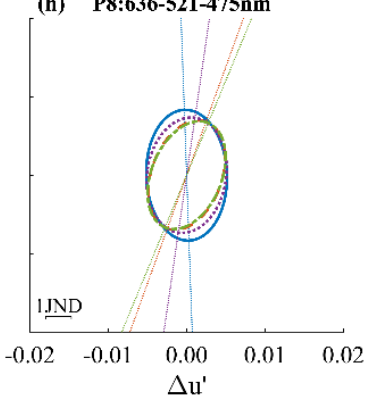

SD ellipse(CIE $\left.196410^{\circ}\right)$ : inter-variability

Fig. 2 Inter-observer variability between the four CMF sets, characterized by the SD-ellipses centered on the origin and grouped per primary set. To visualize differences in ellipse orientation, a line drawn through the origin along the maximal eigenvector of the ellipse covariance matrices is also plotted. The SD-ellipses for different CMFs are indicated by different colors.

The effect of the within-subject factors ('primary set' and 'CMF set') on the observer variability can be assessed by the changes in the size, shape and orientation of the SD ellipses in Figs. 2. Their numerical details are given in Table 1.1 in the Supplemental Material.

From Fig. 2 and Table 1.1, several observations can be made with regard to interobserver variability. Firstly, it can be seen that the ellipses of matches made with sets containing both the $521 \mathrm{~nm}$ and the $447 \mathrm{~nm}$ primaries (P3, P5, P7) have a relatively smaller size, which indicates a smaller inter-observer variability. Similar results have been found by (Hu et al. 2020), where the primary sets containing $525 \mathrm{~nm}$ and $450 \mathrm{~nm}$ primaries were found to provide the smallest matching variability. Secondly, for those primary sets, increasing the peak wavelength of the red primary from $598 \mathrm{~nm}$ to $636 \mathrm{~nm}$ to $673 \mathrm{~nm}$ progressively reduces the inter-observer variability along the $v$ '-direction (see Figs. 2c, 2b, 2d, respectively). Thirdly, in general, switching from a $521 \mathrm{~nm}$ to a $502 \mathrm{~nm}$ green primary causes a slight increase in the 
inter-observer variability along the $u$ '-direction (see Figs. $2 \mathrm{a}$ versus $2 \mathrm{~b}$ and $2 \mathrm{e}$ versus $2 \mathrm{f}$ ). Furthermore, switching the $447 \mathrm{~nm}$ blue primary to a $475 \mathrm{~nm}$ one, causes an increase in the inter-observer variability along the $v^{\prime}$-direction, while keeping the ellipse orientation stable for the $10^{\circ} \mathrm{CMFs}$, but causing a rotation to a near-vertical orientation for the $2^{\circ} \mathrm{CMFs}$. Finally, the blue $404 \mathrm{~nm}$ (P2, P4, P6) primary causes an even larger inter-observer variability along the vertical direction with an overall vertical orientation for all CMF sets. Increased inter-observer variability was also reported by Asano (Asano 2015) for matches made with a $400 \mathrm{~nm}$ primary. A possible reason for the increased variability could be large individual differences in the density of the macular pigment or lens. It confirms Abraham's finding that the magnitudes of dispersions of the matching chromaticity were consistent with lens transmission (Ábrahám et al. 1995).

The general findings on inter-variability discussed above were also statistically verified by checking the homoscedasticity of the variance-covariance matrices using Box's M-test (Box 1949). Multivariate normality is required for this statistical test. As numerical multivariate normality tests, such as Mardia's test for multivariate skewness and kurtosis, tend to become too restrictive for large sets (Field et al. 2012), the multivariate normality of data was graphically confirmed using Quantile-Quantile plots (QQ-plots). More details and typical QQplots for the matching data can be found in the Appendix of PART I.

The results of Box's M-test show that, firstly, no significant differences between the inter-observer variability (covariance matrices) of the four different CMFs sets could be detected for any of the primary sets, except for P6 $(p=0.049)$ between the CIE 1931 and CIE $20062^{\circ}$ sets (see Table 2.1 in the Supplemental Material for the other $p$-values). After Bonferroni correction, P6 also becomes non-significant. These results are consistent with their, generally, similar values for the ellipse orientation angle, area and lengths of the major and minor axis in Table 1.1 and becomes especially clear in Fig. 2. Significant differences in variability, even after a Bonferroni correction, were however found between the various primary sets and this for each CMF set (all $p<0.0001$ ), which confirms the dissimilar ellipses in Figs. 2. Pairwise Box M-tests between each primary set (contrasts) were therefore performed for each CMF set. Results (given in Table 2.2 in the Supplemental Material) indicate that all primary set contrasts were significant, except for the following 10 pairs: P13, P17, P24, P26, P28, P35, P37, P46, P57, P78. In addition to these, the CIE $19312^{\circ} \mathrm{CMFs}$ had 5 additional non-significant pairs (P12, P18, P23, P27, P48, P68); the CIEPO06 $2^{\circ} \mathrm{CMFs}$ had 1 (P38) and both the $10^{\circ} \mathrm{CMFs}$ had 4 (P12, P18, P38, P58). Looking more closely at the type of peak wavelength contrasts these primary set pairs represent, shows that they are nearly all pairs that do not involve a switch to or from a $404 \mathrm{~nm}$ primary, with the exception of one or two (P28 and P12) for the $196410^{\circ}, 20062^{\circ}$ and $200610^{\circ} \mathrm{CMFs}$. On the other hand, the CIE $19312^{\circ}$ CMF set has six such primary set pairs, indicating that its variability when predicting matches is less affected by the peak wavelength of the short-wavelength matching primary, while the other CMF sets do have an increased variability for primary sets with a $404 \mathrm{~nm}$ peak wavelength, as can be clearly observed in Fig. 2. Not surprisingly, these are the same primary sets where significant differences between the mean chromaticity values of younger and older test subjects were found in PART I (see also the SD ellipses in Fig. 3). This suggests that the larger variability for these primaries is due to the presence of two heterogeneous groups (younger/older) whose cone fundamentals or color matching functions react differently to the 
specific peak wavelengths of the blue matching primary, thereby causing additional variability. That for these $404 \mathrm{~nm}$ containing primary sets, the inter-observer variability of the older participant group overlaps substantially with that of the younger groups (see Figs. 3 e,f,g) indicates that a good portion of the older observers have CMFs similar to those of the younger group. In other words, some 'older' observers have 'young' CMFs, which confirms findings by Sarkar (Sarkar, Blonde, et al. 2009) that eye age is not necessarily identical to the actual observer age.

Significant differences in variability between the groups of all between-subject factors 'age' $(p<0.0001)$, 'gender' $(p<0.0001)$, 'ethnicity' $(p<0.0001)$ and 'eye color' $(p=0.0012)$ could be detected using a series of Box M-tests when pooling all factors. Looking into group contrasts for each between-subject factor per level of each of the within-subject factors the following was found. Firstly, for 'age', significant variability between the factor levels could be detected for primary sets P5, P6, P8 for all CMF sets, with additionally P4 for the CIE 1931 $2^{\circ} \mathrm{CMFs}$ (with the $p$-values for the other CMFs barely missing the 5\% significance level by less than 0.5\%). After a Bonferroni correction, only P5 remained significant. Secondly, for 'ethnicity', P3, P5 and P6 were significant, with only P5 remaining after correction. Finally, for the factors 'gender' and 'eye color', respectively only P5 and P7 were significant. No significance remained after a Bonferroni correction. Overall it can be concluded that substantial amounts of significant contrasts could only be detected for the primary set factor, while for the other factors there were no or few such cases.
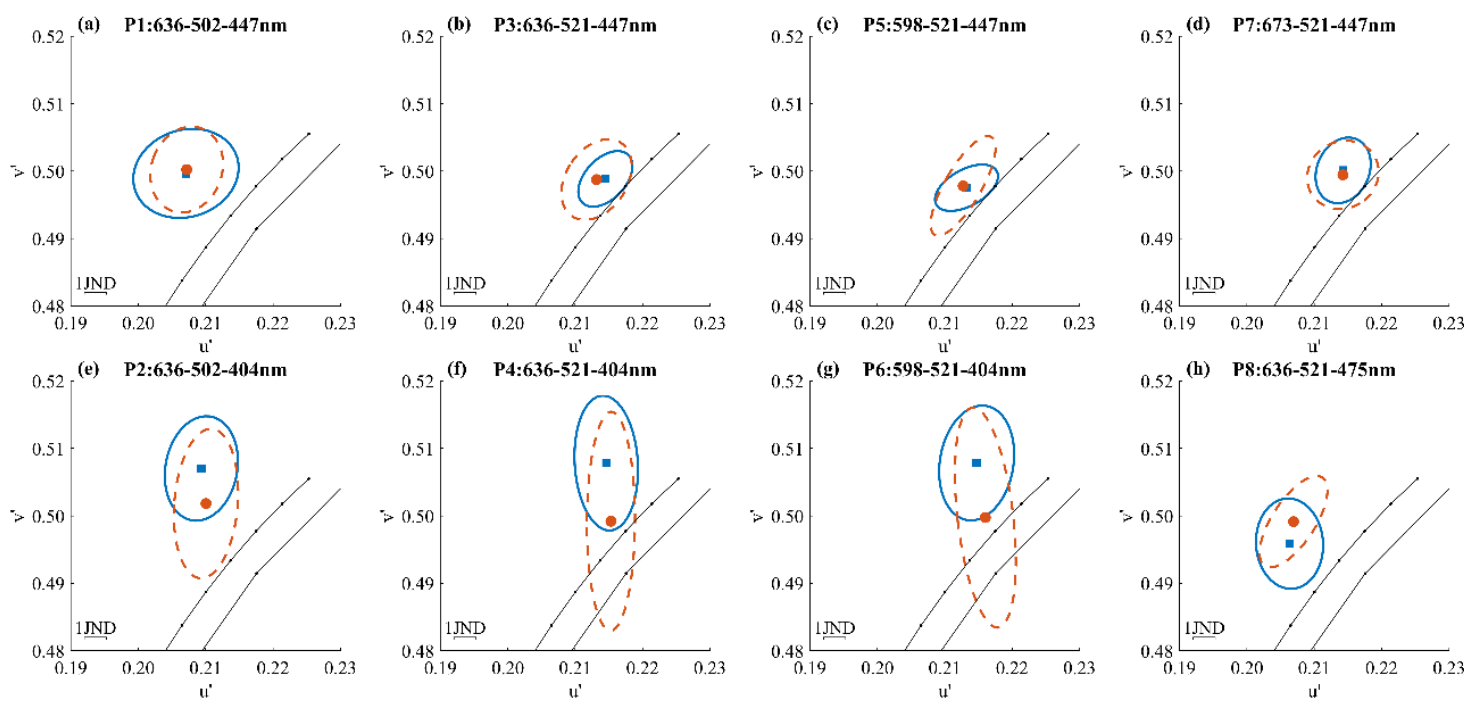

Fig. 3: The SD ellipses (colored solid lines) for matches made by younger (blue) and older (red) observers and the mean chromaticities calculated by CIEPOO6 $10^{\circ} \mathrm{CMFs}$ plotted separately for each primary set (graphs ah). The two black lines represent respectively the blackbody and daylight locus.

\subsection{Intra-observer variability}

The intra-observer variability SD-ellipses (for both the average observer and the individual observer), obtained from the four match chromaticities for each of the four starting points, and computed by 4 CMF sets, are shown in Fig. 4. 

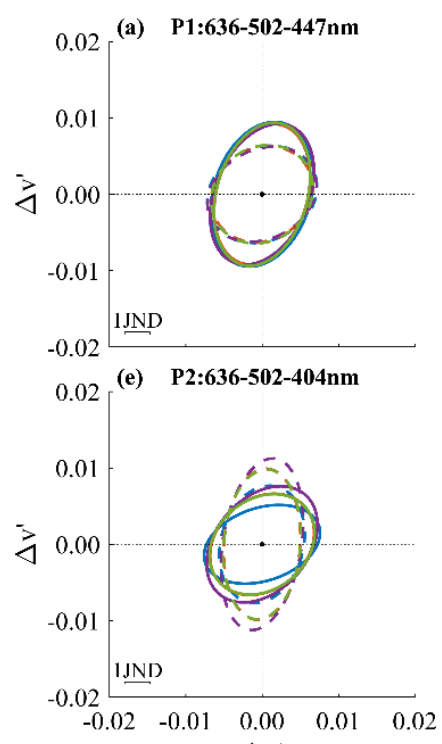

$\Delta \mathrm{u}^{\prime}$

- SD ellipse(CIE $\left.196410^{\circ}\right)$ : intra-variability - - SD ellipse(CIEPO06 $2^{\circ}$ ): inter-variability - - - SD ellipse(CIEPO06 10 $10^{\circ}$ ): inter-variability

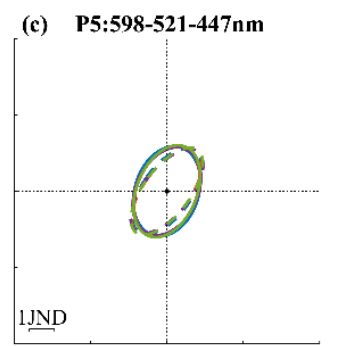

): intra-variability - SD ellipse(CIEPO06 10 $0^{\circ}$ ): intra-variability (g) P6:598-521-404nm

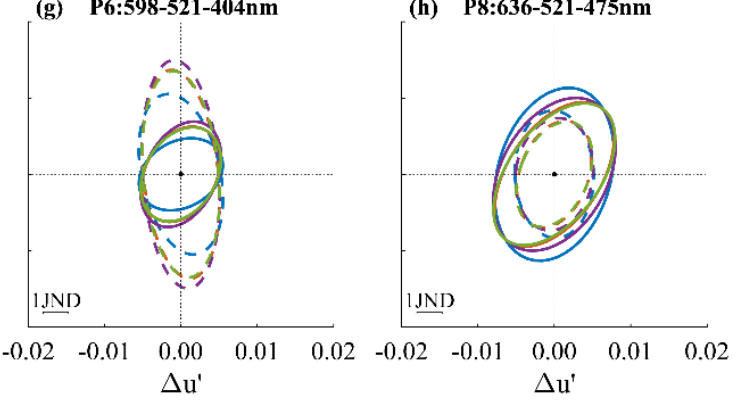

(d) $\quad P 7: 673-521-447 \mathrm{~nm}$

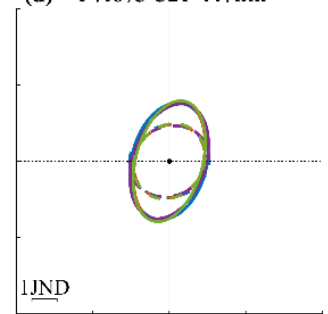

(h) P8:636-521-475nm
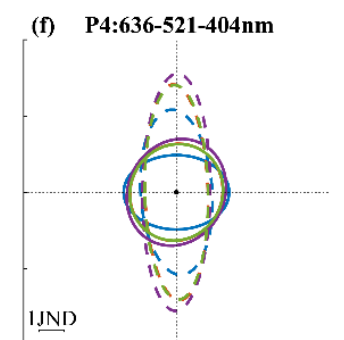

IJND

Fig. 4 - The mean intra-observer variability SD ellipses (solid lines) were plotted for each primary set (subfigures $a-h)$ separately. The inter-observer variability SD ellipses (dashed lines) were also plotted for comparison. All the ellipses are centered on the origin. The SD-ellipses for different CMFs are indicated by different colors.

These intra-variability ellipses are again centered to the same origin for clarity, and grouped per primary set as the latter has been shown to have a substantial impact of match chromaticity (see PART I). In other words, these SD-ellipses represent the match precision a single observer can obtain for a specific primary set. For comparison, the inter-observer variability ellipses were also plotted and centered to the same origin. The inter-observer ellipses are calculated based on the means of matches from 4 starting points for 54 observers, which therefore exclude intra-variability.

From Fig. 4, some observations can be made. First, it is clear that the two $10^{\circ} \mathrm{CMFs}$ result in very similar intra- and inter-observer variability for each primary set, which is understandable as these two CMF sets are very similar. Similar good agreement was also reported for the mean matches predicted by these sets in PART I. For intra-variability, the CIEPO06 $2^{\circ}$ set shows quite similar ellipse sizes and orientations (both for inter- and intravariability) with those of the $10^{\circ} \mathrm{CMFs}$ for the primary sets containing the $447 \mathrm{~nm}$ blue primary (P1, P3, P5 and P7). However, the primary sets containing peaks at $404 \mathrm{~nm}(\mathrm{P} 2, \mathrm{P} 4, \mathrm{P} 6)$ and $475 \mathrm{~nm}$ (P8) are slightly different. Specifically, the SD ellipses computed with the CIEPO06 $2^{\circ} \mathrm{CMFs}$ are slightly rotated compared to those of the $10^{\circ} \mathrm{CMFs}$ for the intra-variability. The inter-variability SD ellipses for CIEPO06 $2^{\circ} \mathrm{CMFs}$ are also slightly tilted compared to the $10^{\circ}$ CMFs for the three primary sets containing $404 \mathrm{~nm}$ (P2, P4, P6), and are, additionally, stretched along the $v^{\prime}$ direction (as can also be seen in Fig. 2). Similar observations can be made for the ellipses based on the CIE $19312^{\circ} \mathrm{CMFs}$. The CMF sets can have a different impact on intraobserver variability than inter-observer variability.

Second, assuming the LED primaries don't have an impact on intra-observer variability, the SD ellipses should remain similar in size, shape and orientation across the eight primary 
sets. However, from Fig. 4, it is clear that the mean intra-variability ellipses show different sizes and shapes for different primary sets. For P1, P3, P7 and P8, the intra-observer variability ellipses are slightly larger than those for other primary sets. The larger intra-observer variability could be due to a reduced matching precision (ability) or due to poorer color discrimination for these specific primary sets. Differently, for primary set P2, P4 and P6, the intra-observer variability tends to be smaller than the inter-observer variability, which is primarily elongated along the $v$ ' direction. The increased inter-observer variability is, as discussed earlier, likely caused by the various degrees of lens-yellowing due to the observers substantially different ages, but this, of course, would not have an impact on the matching ability of a single observer. Different primary sets, therefore, can have a different impact on intra-observer variability than inter-observer variability.

\subsection{Comparison with CIE deviate observer (1989), Asano (2015) observer variability}

The inter-observer variability obtained in the experiment was compared with those predicted by the CIE standard deviate observer (CIE 1989), and that obtained in Asano's (Asano 2015; Asano, Fairchild, Blondé, et al. 2016) work. For the latter, a comparison was also made for the intra-observer variability.

\subsubsection{Comparison with CIE deviate observer (1989)}

Inter-observer variability was assessed using the CIE standard deviate observer with the broadband reference spectrum (target) as a stimulus spectrum and also with calculated narrowband spectra of the same reference chromaticity and composed of the same primaries as the match field. The latter were also determined as in the experiment, an observer had to match a broadband reference field with a narrowband match field, both of which are expected to exhibit different variabilities. The results are shown in Fig. 5. The mean matches made with each primary set by all the observers in the current experiment are shown in the figure, as well as their SD ellipses.

Unlike the actual observer matches made with narrowband primaries, the predicted deviate observer matches for the broadband reference spectrum change very little across CMF sets. As found in PART I (Li et al. 2020), the smooth broadband spectrum resulted in very similar chromaticity values for all CMF sets. However, for each CMF set and primary set, the deviate observer predictions for the calculated narrowband spectra were larger, but still much smaller than the mean observer matches in the current experiment. For the CIE $19312^{\circ} \mathrm{CMFs}$, the underestimation by the CIE standard deviate observer was strongest, confirming earlier reports by other researchers (North and Fairchild 1993; Rich and Jalijali 1995; Alfvin and Fairchild 1997; Sarkar, L. Blondé, et al. 2010). Furthermore, it can be observed that the deviate observer chromaticity predictions for the simulated spectra composed of different primary sets have very similar values, which are actually spread around that of the spectrally broadband reference. Note that for the stable set P3 (636-521-447 nm), the observer matches in the current study have smaller variability, but still, a strong underestimation by the CIE standard deviate observer was found. 
The underestimation for narrowband primary sets by the CIE deviate observer, was confirmed by calculating the standard deviate observer responses based on the mean match made with each primary set, assuming that the mean match represents the actual matching target perceived by observers when looking at their match field under this specific primary set. The results are shown in Fig. 6, where for each CMF set and for each primary set, the SD ellipses of the actual matches and the match coordinates calculated using the CIE standard deviate observer are plotted. Even in this case, which ignores the impact of matching narrowband primaries to a broadband reference, the variability predicted by the CIE standard deviate observer is still substantially smaller especially for P4 and P6. In addition, the latter have the smallest predicted deviate observer variability, while their actual inter-variability is the largest among all the primary sets. A similar, but less pronounced, tendency can be found for P2. As mentioned in section 3.1, the larger inter-variability found for these three primary sets, which include a $404 \mathrm{~nm}$ primary, is likely caused by substantial differences in the lens density of the observers. The CIE standard deviate observer variability estimate was substantially larger even for the stable P3 set (636-521-447 nm), which has peak wavelengths close to those of the Stiles \& Burch (Stiles and Burch 1955b) matching primary set (645-526$444 \mathrm{~nm}$ ) from which the deviate observer was derived (Nayatani et al. 1985; Takahama et al. 1985).

The standard deviate observer gave the most accurate, but still underestimated, prediction of inter-variability for $\mathrm{P} 8$ and the $10^{\circ} \mathrm{CMFs}$.
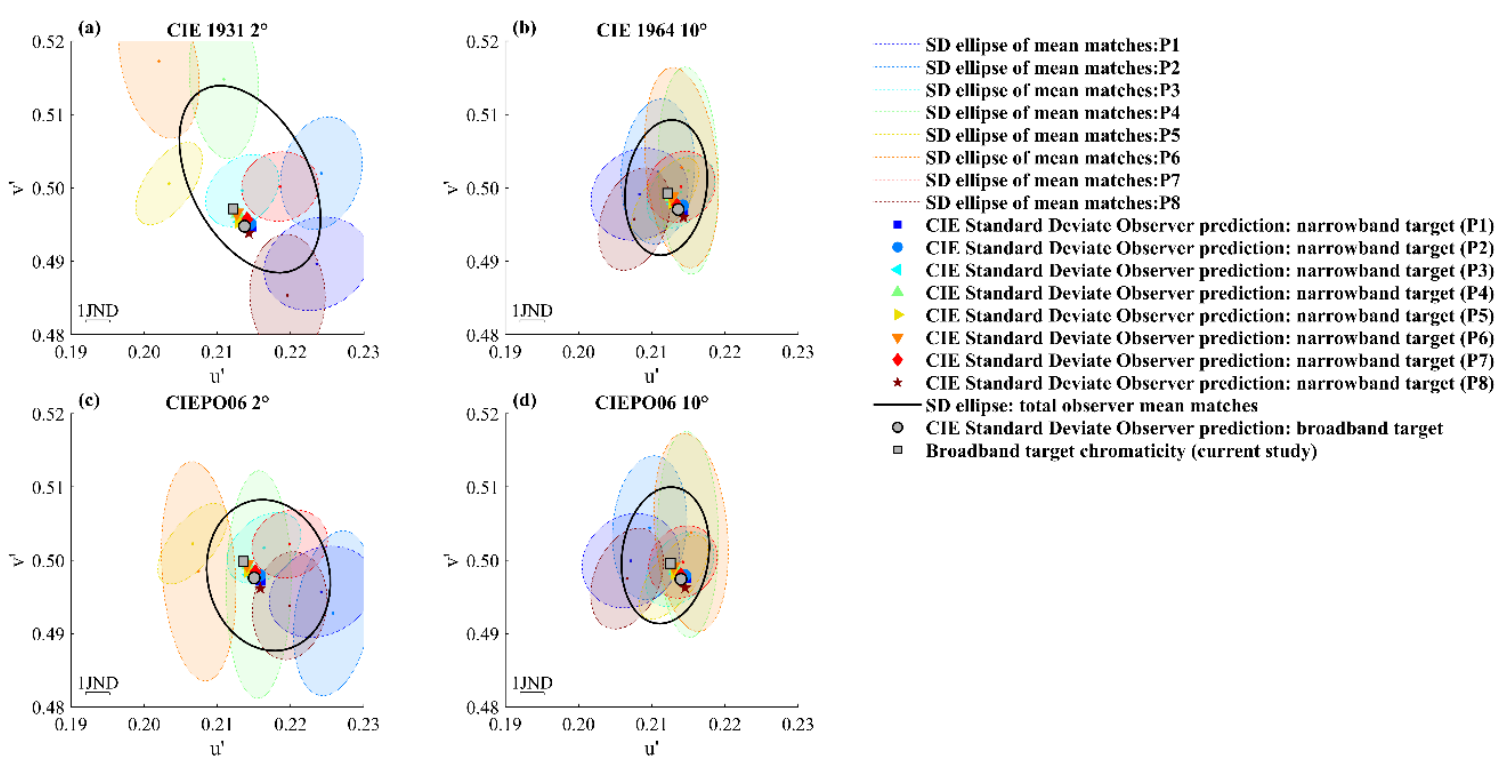

Fig. 5 - The overall inter-observer variability SD ellipse (black solid line) in the current experiment, the matches made by observers grouped per primary set and their SD ellipses (different colored dots and color filled ellipses), the chromaticity of the broadband matching target in the experiment (grey filled square), as well as the CIE standard deviate observer match predictions for the spectrally broadband target (grey filled circle) and for the spectrally narrowband simulated target (color filled markers with different shapes) are plotted for the four different CMF sets: CIE $19312^{\circ}$, CIE $196410^{\circ}$, CIEPOO6 $2^{\circ}$, and CIEPOO6 $10^{\circ}$. 


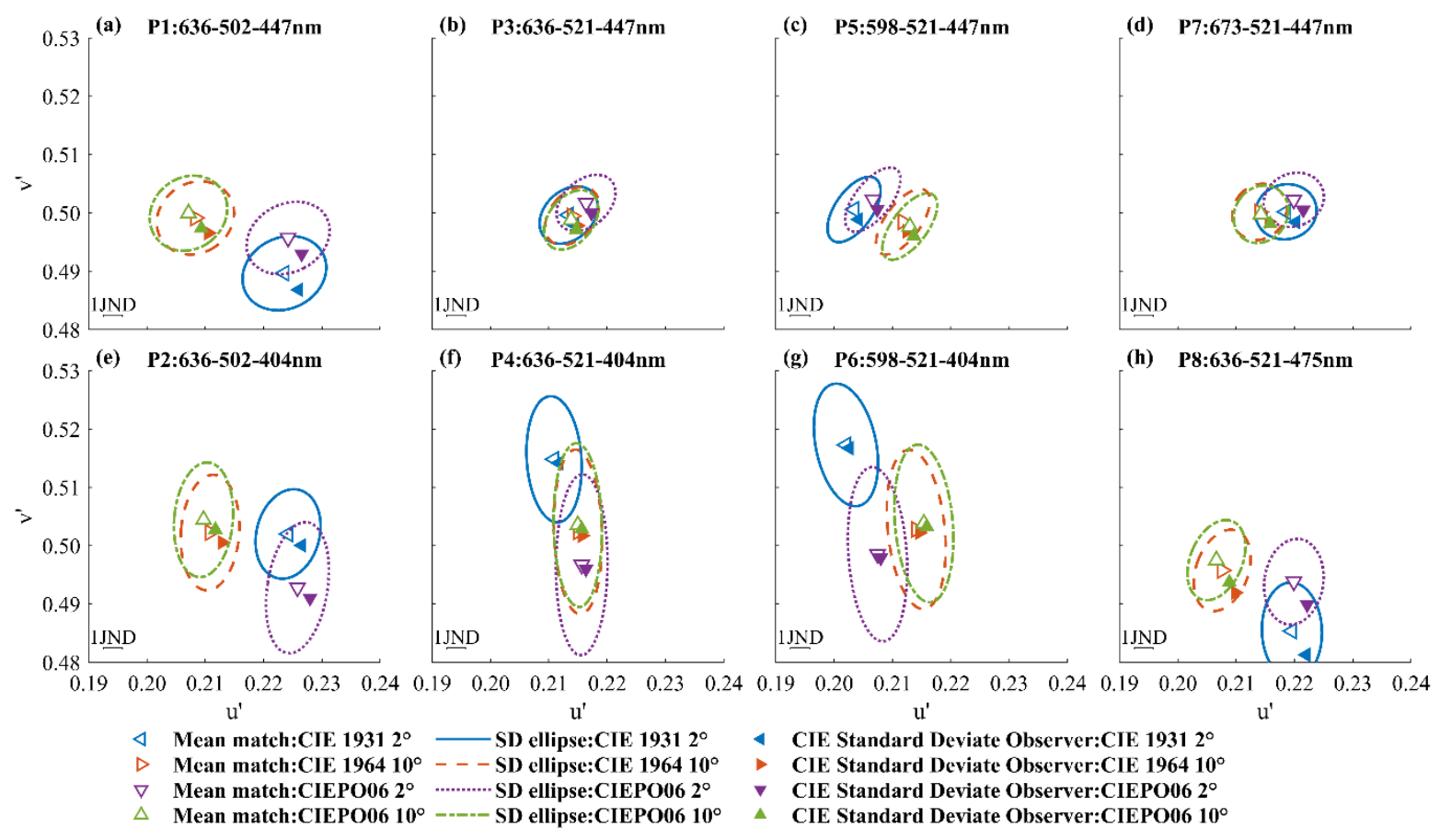

Fig. 6-Mean CIE 1976 u'v' chromaticity of matches (empty markers with different shapes) and their SD ellipses for different primary sets (subplots $a-h$ ) and different CMF sets. The color filled markers represent the CIE standard deviate observer matches corresponding to the mean matches calculated for the four different CMF sets (Blue: CIE $19312^{\circ} \mathrm{CMFs}$, Red: CIE $196410^{\circ} \mathrm{CMFs}$, Purple: CIEPO06 $2^{\circ} \mathrm{CMFs}$, Green: CIEPO06 $10^{\circ} \mathrm{CMFs}$ ).

\subsubsection{Comparison with observer variability in Asano's color matching experiment (2015)}

The inter-observer and intra-observer variability of matches made by the observers from the current study were compared with that found for the observers in a 2015 study by Asano(Asano 2015). In that experiment, matches were made to two colored and three neutral reference stimuli. Two of the latter were phosphor-white LEDs representing a spectrally broadband spectrum, much like in our study. The matches to the two neutral spectrally broadband reference stimuli, referred to as 'Match 1' and 'Match 2', were made by a group of 151 observers using two different narrowband primary sets at a luminance of $25 \mathrm{~cd} / \mathrm{m}^{2}$. The LED matching primaries for 'Match 2' (592-521-400 nm) were very similar to one of our matches, i.e. P6 (598-521-404 nm). 'Match 1':657-506-469 nm was somewhat similar to our P1 (636502-447 nm) and P8 (636-521-475 nm) primary sets. However, because of the larger impact of the blue primary set on inter-observer variability (see earlier) and the substantially smaller difference of the blue primary in P8 with that in Asano's 'Match 1' primary set, P8 was selected for further comparison.

The individual mean matches for P8 and 'Match 1', P6 and 'Match 2' and their interobserver variability SD ellipses were computed and plotted in Figs. 7a,d. Because of the different match references used in the two experiments, the two groups of matches are clearly separated, no overlap between ellipses from different experiments can be observed. To visualize the distances between observer matches and the matching target in each experiment, the targets in both experiments were centered to the same origin, keeping the relative locations of their corresponding SD ellipses, as shown in Figs. 7b,e. To better compare the observer 
variability, the center of all the ellipses were shifted to the origin in Figs. 7c,f. It can be seen from Figs. 7a,d that there are very small differences between the chromaticity computed by the four CMF sets for the spectrally broadband reference, though for Asano's somewhat more spectrally structured references, there are substantial differences between the chromaticity values calculated with $2^{\circ}$ and $10^{\circ} \mathrm{CMFs}$. On the other hand, the relative locations and dispersions of the SD ellipses computed by the four CMF sets are quite similar for both experiments. Asano's observers did tend to match less accurately than the observers in this study, especially when comparing 'Match 1' and P8 (see in Figs. 7b). From Figs. 7c,f, it can be noticed that in both comparisons, Asano's observers tend to have a larger inter-variability than the observers from the current study, especially when comparing 'Match 1' with P8. Note that the ellipses of 'Match 1' are expanded horizontally from those of P8, and the ellipses of 'Match 2' are vertically larger than those for P6, but only slightly. A possible cause is the difference in the peak wavelengths of the primaries of P8 and 'Match 1'. Although the blue primaries in the two sets are very similar (only $6 \mathrm{~nm}$ difference), the green primary was more dissimilar (19 $\mathrm{nm}$ difference), as was the red primary $(21 \mathrm{~nm})$. As discussed in section 3.1, changing the green primary from $521 \mathrm{~nm}$ (in P8) to $502 \mathrm{~nm}$ (in 'Match 1') would cause a slight horizontal expansion of the SD ellipse as can be seen by comparing Figs. $2 b$ and $2 a$ and by comparing Figs. $2 f$ and 2e. Earlier, the impact of progressively increasing the peak wavelength of the red primary from $598 \mathrm{~nm}$ to $636 \mathrm{~nm}$ to $673 \mathrm{~nm}$ was also discussed. The intermediate peak wavelength of $657 \mathrm{~nm}$ of the Asano red primary would, therefore, result in a slight decrease along the $v$ '-direction compared to the $636 \mathrm{~nm}$ peak wavelength of the primary in our study.

Importantly, the overall smaller inter-variability found for our matches could also due to the different methodology that was used in the experiments. Although no details seem to be given anywhere on the exact procedure of obtaining the repeat matches in Asano's work (Asano 2015; Asano, Fairchild, Blondé, et al. 2016), they do seem to follow much of the procedures established by Sarkar (Sarkar, Laurent Blondé, et al. 2010). In Sarkar's experiments, the initial chromaticity for each repeat match was each time set to the same hue and lightness values with the chroma randomly varied between $75 \%$ and $90 \%$ of their target chroma (Sarkar, Laurent Blondé, et al. 2010). In this case, the three repetitions would lead to a smaller intra-observer variability and a bias of the matches for each observer compared to the current study, which adopted a four-starting-chromaticity method with starting hues equally distributed along the hue circle and with high chroma to minimize biasing the final match and allowing for a more realistic estimate of the observer variability. In addition, possible rod intrusion, as the Asano experiments were performed under dark-adapted conditions with a match luminance of around $25 \mathrm{~cd} / \mathrm{m}^{2}$, could also have led to a larger inter-observer variability. 

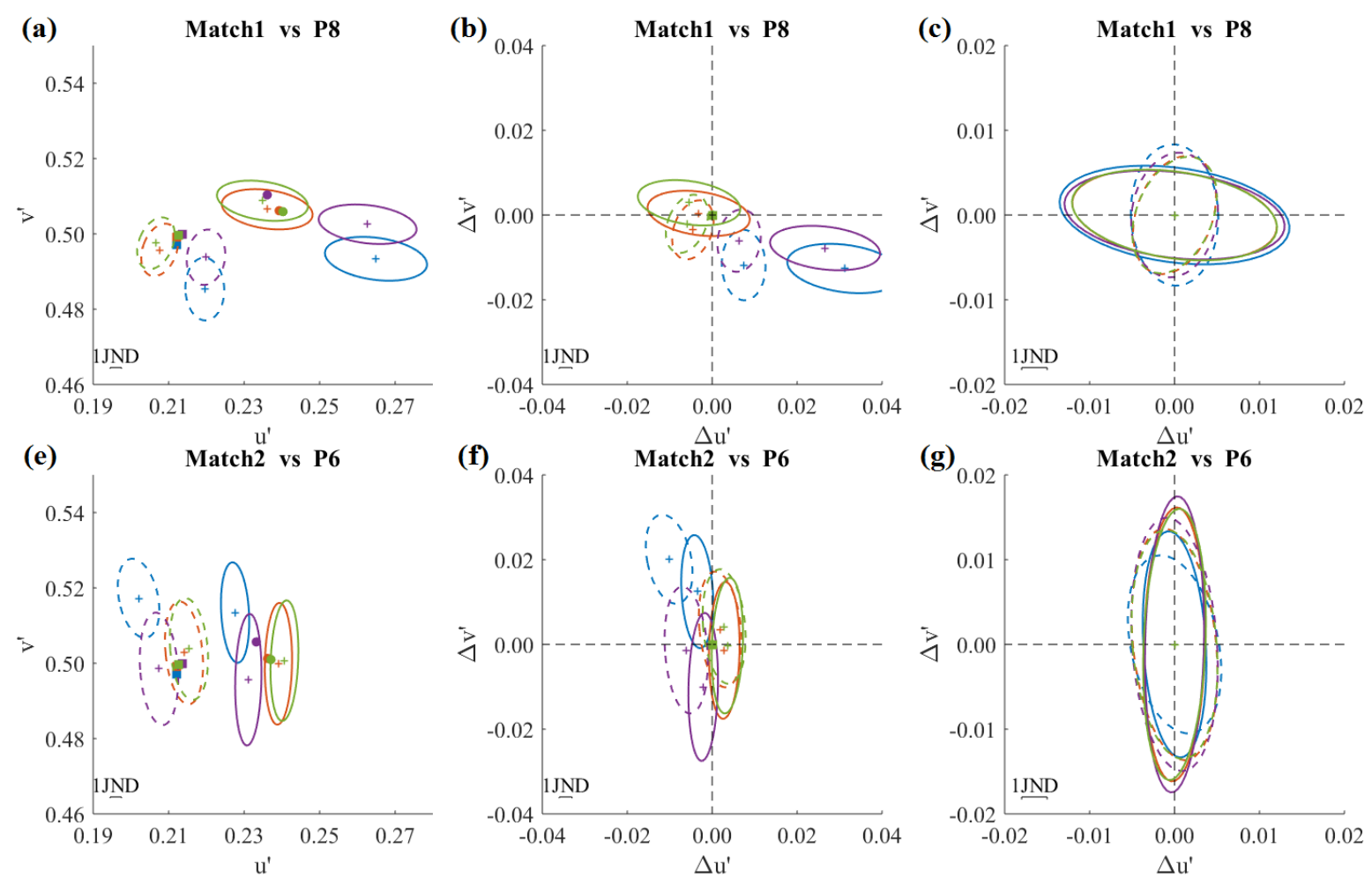

$$
\begin{aligned}
& \text { - Matching target: CIE } 19312^{\circ} \text { (Asano's study) - Matching target: CIE } 196410^{\circ} \text { (Asano's study) } \\
& \text { - Matching target: CIE } 19312^{\circ} \text { (current study) - Matching target: CIE } 196410^{\circ} \text { (current study) } \\
& \text { SD ellipse: CIE } 19312^{\circ} \text { (Asano's study) - SD ellipse: CIE } 196410^{\circ} \text { (Asano's study) } \\
& \text { - - - - SD ellipse: CIE } 19312^{\circ} \text { (current study) - - - - SD ellipse: CIE } 196410^{\circ} \text { (current study) } \\
& \text { - Matching target: CIEPO06 } 2^{\circ} \text { (Asano's study) - Matching target: CIEPO06 } 10^{\circ} \text { (Asano's study) } \\
& \text { - Matching target: CIEPO06 } 2^{\circ} \text { (current study) Matching target: CIEPO06 } 10^{\circ} \text { (current study) } \\
& \text {-SD ellipse: CIEPO06 } 2^{\circ} \text { (Asano's study) - SD ellipse: CIEPO06 } 10^{\circ} \text { (Asano's study) } \\
& \text { - - - SD ellipse: CIEPO06 } 2^{\circ} \text { (current study) - - - SD ellipse: CIEPO06 } 10^{\circ} \text { (current study) }
\end{aligned}
$$

Fig. 7 - Mean chromaticity coordinates of matches (color crosses) and their SD ellipses (dashed lines) for P8, P6 in the actual experiment and our matching targets (color squares), mean matches (color crosses) and their SD ellipses (solid lines) for 'Match 1' and 'Match 2' made in Asano's experiment and their matching targets (color dots) are compared respectively in subplots a,d. To better visualize location differences of matches made in two experiments, the matches in both experiments are centered on the same origin (matching target), keeping the relative distances between matching ellipses and their corresponding targets. The ellipses are plotted in subplots $b$,e. To better compare the dispersion of matches, all the SD ellipses are centered on the origin (mean of the matches corresponding to each ellipse) in subplots c,f. The SD-ellipses for different CMFs are indicated by different colors.

\subsection{Inter-observer variability and accuracy of Asano's 10 \& Sarkar's 8 Categorical} Observers for a $10^{\circ}$ field of view

The chromaticity of the matches made by the observers in the current study were calculated using the CMF sets of Asano's (Asano and Fairchild 2020) and Sarkar's (Sarkar 2011b) Categorical Observers for a $10^{\circ}$ stimulus field size. Asano's 10 Categorical Observers are sorted in terms of their representativeness of the population. The first catgeorical observer is equivalent to the CIEPO06 $10^{\circ} \mathrm{CMFs}$ with the age parameter changed to 38 years, the other categorical observers have varying values for the physiological parameters in the Asano Individual Observer Model. Sarkar's reduced set of 8 observer categories were derived from the Stiles \& Burch matching data, and ordered from the least to the most representative for that set. The last and the most representative set of Sarkar's Categorical Observers is the CIE 1964 
$10^{\circ}$ observer. However, in a color matching study with 49 observers at two locations (Germany and Hungary) Sarkar (Sarkar 2011b)found that the CIE $10^{\circ}$ observer accounted for only $8 \%$ of the observer's matching results. The three most representative categorical observers accounted for 25\% (CAT-4), 20\% (CAT-5) and 18\% (CAT-6) (Sarkar 2011b).On the other hand, in another study (Sarkar, L. Blondé, et al. 2010), the most representative categorical observers were CAT-7 (30\%) and CAT-5 (27\%), with the latter being somewhat close the CIE $196410^{\circ}$ observer. The CIE $196410^{\circ} \mathrm{CMFs}$ result in similar match predictions to the CIEPO06 $10^{\circ}$ CMFs, as can be seen from Fig. 2 and Fig. 5. The calculated chromaticities and their SD ellipses using these two categorical observer sets are shown respectively in Fig. 8 and Fig. 9, the ellipses for CIEPO06 $10^{\circ} \mathrm{CMFs}$ were also indicated for comparison.

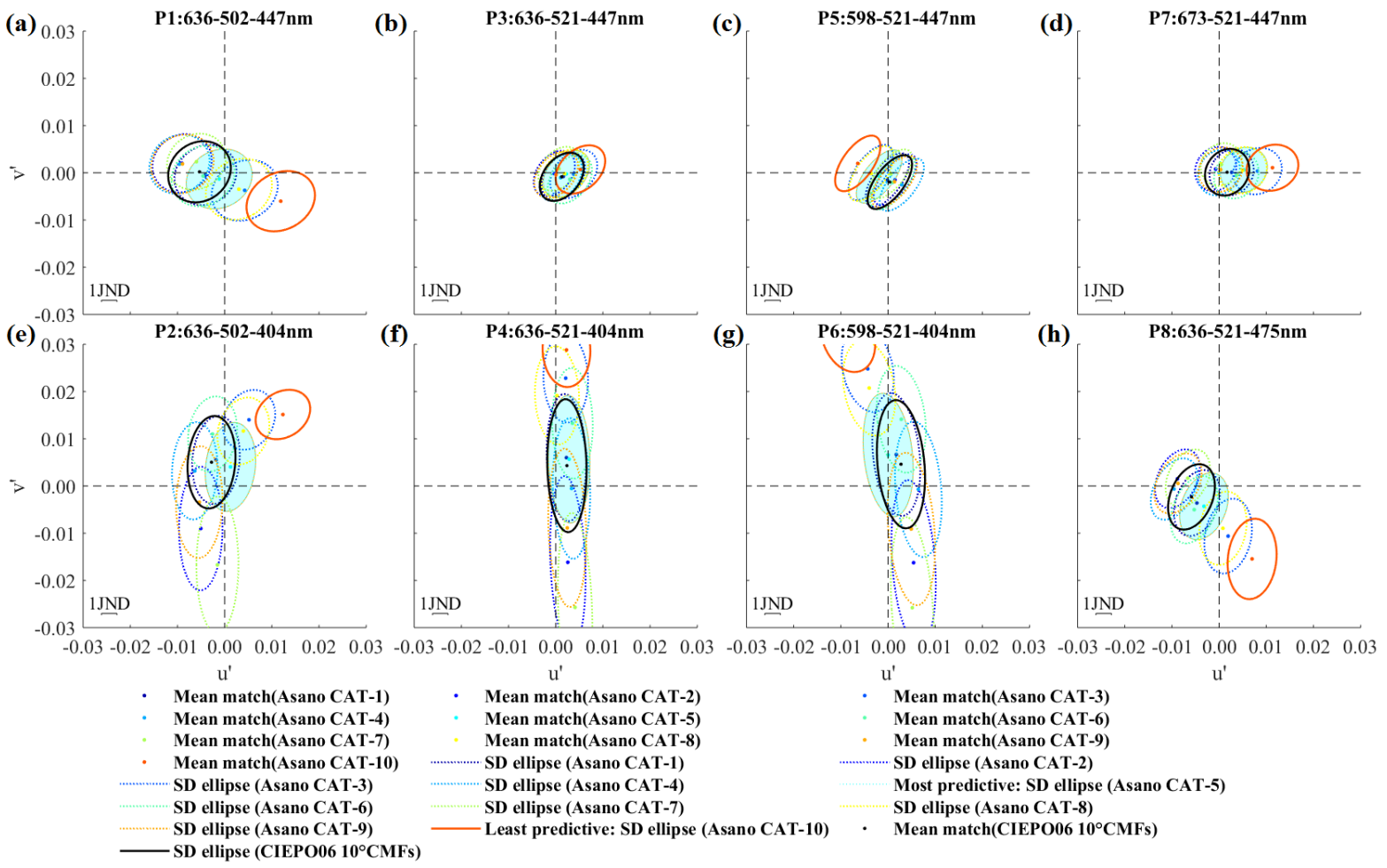

Fig. 8 - Mean chromaticity coordinates of matches (color dots) and their SD ellipses (dotted lines) calculated using Asano's Categorical Observers, are plotted for different primary sets (subplots $a-h$ ). To better visualize the location differences of matches, the matches for all the CMF sets are centered on the same origin (target chromaticity), keeping the relative distances between ellipses and their corresponding targets. The ellipses calculated using CIEPOO6 $10^{\circ} \mathrm{CMFs}$ (black lines) were also plotted for the comparison. The SD-ellipses of the most predictive categorical observer are indicated with filled face color, and those of the least predictive one are shown by solid red lines. 

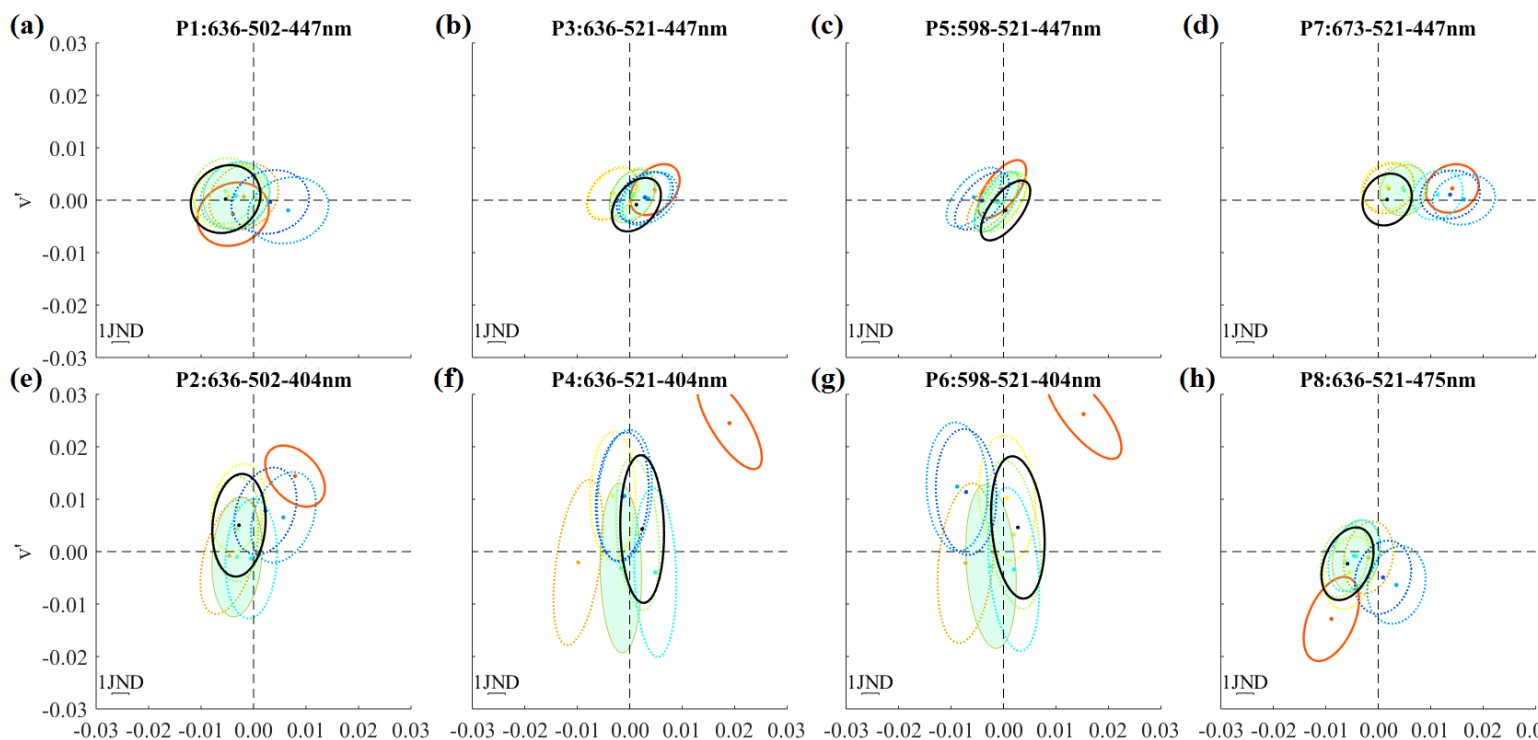

P6:598-521-404nm

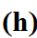

(h)

P8:636-521-475nm

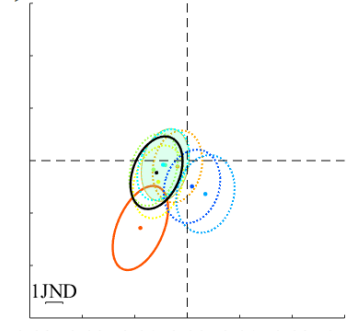
$\mathrm{u}^{\prime}$

Mean match(Sarkar CAT-1)
Mean match(Sarkar CAT-5)
Least predictive: SD ellipse (Sarkar CAT-1)
Most predictive: SD ellipse (Sarkar CAT-5)

Mean match(Sarkar CAT-2) Mean match(Sarkar CAT-6) DD llipse (Sarkar CAT-2)

SD ellipse (Sarkar CAT-6) Mean match(CIEPO06 $10^{\circ} \mathrm{CMFs}$ )

Mean

SD ellipse (Sarkar CAT.

SD ellipse (Sarkar CAT-7)

$\mathrm{u}$

Mean match(Sarkar CAT-4)

Mean mate(Sarkar CAT-8)

SD ellipse (Sarkar CAT-4)

SD ellipse (Sark CAT-8)

Fig. 9 - Mean chromaticity coordinates of matches (color dots) and their SD ellipses (dotted lines) calculated using Sarkar's Categorical Observers, are plotted for different primary sets (subplots a-h). To better visualize the location differences of matches, the matches for all the CMF sets are centered on the same origin (target chromaticity), keeping the relative distances between ellipses and their corresponding targets. The ellipses calculated using CIEPOO6 $10^{\circ} \mathrm{CMFs}$ (black lines) were also plotted for the comparison. The SD-ellipses of the most predictive categorical observer are indicated with filled face color, and those of the least predictive one are shown by solid red lines.

From Fig. 8, the most predictive categorical observers in the current study is the Asano CAT$5 \mathrm{CMF}$ set in terms of accuracy $\left(\Delta u^{\prime} v^{\prime}=0.0042\right.$, slightly larger than $\left.1 \mathrm{JND}\right)$ and variation of matches (Major ellipse axis $=0.0043 u$ 'v' units). The average CIE $1976 u$ 'v' chromaticity difference (over all primary sets) between the mean matches (over all observers) of Asano CAT-5 and the target chromaticity (also called matching inaccuracy) is almost identical $\left(<10^{-}\right.$ ${ }^{4} u$ 'v' units) with that for the CIEPO06 $10^{\circ}$ standard average observer. For primary set P1, the matches predicted by Asano CAT-5 are slightly closer to the target, while the opposite is the true for P7. The dispersions of the SD ellipses for the Asano CAT-5 and the CIEPO06 $10^{\circ}$ CMFs are also similar.

Sarkar's CAT-5 observer is also found to be the most predictive one among the eight categorical observers, in close agreement with the Sarkar's findings of CAT-5 being one of the top representative categories (Sarkar, L. Blondé, et al. 2010; Sarkar 2011b). Its average matching inaccuracy is similar to or smaller than that of the CIEPO06 $10^{\circ} \mathrm{CMFs}\left(\Delta u^{\prime} v^{\prime}\right.$ ' (Sarkar CAT-5) $=0.003$ vs $\Delta u^{\prime} v^{\prime}\left(\right.$ CIEPO06 $\left.\left.10^{\circ}\right)=0.0041\right)$. However, the matching inaccuracy of Sarakar's CAT-5 is noticeably smaller than that of Sarkar's CAT-8 observer $\left(\Delta u^{\prime} v^{\prime}=0.0071\right)$, also known as the CIE $196410^{\circ}$ observer. The dispersion of the SD ellipses calculated using Sarkar's CAT-5 observer and the CIEPO06 $10^{\circ} \mathrm{CMFs}$ are almost identical (average difference of the ellipses' lengths is smaller than $10^{-3} u$ 'v' units). However, for primary sets such as P4 and P6, the ellipses of Sarkar's CAT-5 observer are slightly larger ( $\geq 10^{-3} u$ 'v' units) than those of the CIEPO06 $10^{\circ} \mathrm{CMFs}$. 
It can be also seen from Figs. 8 and 9 that, the least representative categorical observers in Asano's and Sarkar's studies also predict worst for our matches in terms of accuracy and variation. In addition, for Asano's Categorical Observers, larger spreads of the ellipses, as well as elongation of the ellipses along the $v$ '-direction, can be observed for the primary sets $(\mathrm{P} 2$, P4, P6) containing the $404 \mathrm{~nm}$ primary. These primary sets mainly result in larger ellipse spreads along the $u$ ' and $v^{\prime}$ directions for Sarkar's Categorical Observers.

\section{Conclusion}

This study investigated the inter- and intra-observer variability in a bi-partite $10^{\circ}$ achromatic color matching experiment using different combinations of spectrally narrowband LED primaries. Color matches were made in a specially designed setup under immersive and stable adaptation conditions at $4673 \mathrm{~K}$, by a group of 54 observers with varying age, gender, ethnicity and eye color. The matching target was a spectrally broadband neutral reference $(4613 \mathrm{~K})$ at $70 \mathrm{~cd} / \mathrm{m}^{2}$, which is the same luminance as the matching field.

Firstly, the impact of the within-subject (primary set, CMF set) and between-subject factors (observer age, gender, ethnicity and eye color) on the inter-observer variability was investigated. For the within-subject factors, both the quantitative statistical analyses and more qualitative visual analyses of the match chromaticity values and their SD/SE-ellipses revealed that the primary set has a statistically significant and important impact in terms inter-observer variability. The primary set with peak-wavelengths (636-521-447 $\mathrm{nm}$ ) close to those in 'typical' RGB-primaries widely known and used in industry has the smallest inter-observer variability of all the different primary sets investigated. The CMF selected to calculate the chromaticity has no significant impact on the variability. For the between-subject factors, other significant effects on inter-variability were found for the between-subject factors 'ethnicity', 'gender' and 'eye color' when pooling all other factors. However, post-hoc contrast tests showed that after a Bonferroni correction, to account for the multiple comparisons, that no or few significant effects on inter-variability remained. Only a significant impact of 'age' factor on inter-variability could be detected for P5. This is consistent with shifts expected from ageinduced yellowing of the lens, requiring some of the observers to add additional shortwavelength light to their match. As can be seen from SD ellipses, the difference in mean match chromaticity between the two groups also leads to an increased variability along the $v$ 'direction for these primary sets.

Secondly, primary sets were found to have a different impact on intra-observer variability than on inter-observer variability, because repeat matches would not be influenced by variations in human eye responses to different primary sets (the observer is basically looking at the stimulus with the same eyes on each repeat match). On the other hand, for some primary sets intra-observer variability was larger than average, indicating observers had more difficulty in making the matches, while these primary sets did not seem to have an increased interobserver variability. In addition, the age factor was found to statistically significantly affect the inter-observer variability, while for an individual observer, his real age might not accurately represent his "eye age".

Finally, observer variability was compared with the predictions of the CIE standard deviate observer and with the observer variability in Asano's experiments (Asano 2015). The 
CIE standard deviate observer predictions for both the spectrally broadband reference and the narrowband simulated reference spectra were found to strongly underestimate the interobserver variability for all the CMF sets, especially the CIE $19312^{\circ}$ CMFs. The variability underestimation of the CIE standard deviate observer is substantial for all the primary sets, especially for those containing $404 \mathrm{~nm}$ LED. Furthermore, different dispersions of the interobserver variability SD ellipses were found between those in Asano's work and this study. Possible reasons are the differences in peak wavelengths of the red and green primaries in the primary sets of the two studies but, also differences in the experimental methodology. Although Asano didn't mention details on the procedure for obtaining repeat matches, they do seem to have used a repetition procedure whereby the initial starting chromaticity is quite close to the target and which is only varied along the chroma dimension, possibly leading to biased matches and observer variability. In contrast, in this study, a four-starting-chromaticity method with the initial chromaticity spread equally along the hue circle and at high chroma, was used. In addition, different adaptation conditions were used. The dark-adapted conditions in Asano's study could have resulted in a higher observer variability (Smet et al. 2014). Finally, a higher luminance level $\left(70 \mathrm{~cd} / \mathrm{m}^{2}\right)$ of the reference was used in the current experiment to minimize rod intrusion, in contrast to the $25 \mathrm{~cd} / \mathrm{m}^{2}$ luminance in Asano's experiments.

\section{Disclosure statement}

The author reports no declarations of interest.

\section{Funding details}

The author reports no funding for the preparation of this article.

\section{References}

Ábrahám G, Körösi H, Schanda J, Shapiro AG, Wenzel K. 1995. Anomalies in additive colour matches. Color Res Appl. 20(4):235-244. doi:10.1002/col.5080200406.

Alfvin RL, Fairchild MD. 1997. Observer Variability in Metameric Color Matches using Color Reproduction Media. Color Res Appl. 22(3):174-188. doi:10.1002/(SICI)15206378(199706)22:3<174::AID-COL6>3.0.CO;2-O.

Allen E. 1970. An index of metamerism for observer differences. In: Proceedings of the 1st AIC congress, Color. Vol. 69. p. 771-784.

Asano Y. 2015. Individual Colorimetric Observers for Personalized Color Imaging. Rochester Institute of Technology.

Asano Y, Fairchild MD. 2020. Categorical observers for metamerism. Color Res Appl.

Asano Y, Fairchild MD, Blondé L. 2016. Individual Colorimetric Observer Model. PLoS One. 11(2):e0145671. doi:10.1371/journal.pone.0145671.

Asano Y, Fairchild MD, Blondé L, Morvan P. 2016. Color Matching Experiment for Highlighting Interobserver Variability. Color Res Appl. 41(5):530-539. 
doi:10.1002/col.21975.

Blackwell KT, Buchsbaum G. 1988. The effect of spatial and chromatic parameters on chromatic induction. Color Res Appl. 13(3):166-173. doi:10.1002/col.5080130309. https://doi.org/10.1002/col.5080130309.

Box GEP. 1949. A General Distribution Theory for a Class of Likelihood Criteria. Biometrika. 36(3/4):317-346. doi:10.2307/2332671. http://www.jstor.org/stable/2332671.

Braun KM, Fairchild MD, Alessi PJ. 1996. Viewing techniques for cross-media image comparisons. Color Res Appl. 21(1):6-17.

CIE. 1986. Colorimetry, Second Edition. CIE. 15.2-1986.

CIE. 1989. Special metamerism index: change in observer. CIE. 080-1989.

CIE. 2004. Colorimetry, 3rd Edition. CIE. 015-2004. http://www.cie.co.at/main/freepubs.html.

CIE. 2006. Fundamental Chromaticity Diagram with Physiological Axes - Part I. CIE. 170$1: 2006$.

CIE. 2015. Fundamental Chromaticity Diagram with Physiological Axes -Part 2: Spectral Luminous Efficiency Functions and Chromaticity Diagrams. CIE. 170-2:2015.

CIE. 2018. Colorimetry, 4th Edition. CIE. 015:2018. doi:10.25039/TR.015.2018. http://www.cie.co.at/publications/colorimetry-4th-edition.

Cuttle C. 2008. Lighting by design. Routledge.

Fairchild MD, Wyble DR. 2007. Mean observer metamerism and the selection of display primaries. In: Color and Imaging Conference. Vol. 2007. Society for Imaging Science and Technology. p. 151-156.

Field AP, Miles J, Field Z. 2012. Discovering statistics using R.

Flecy L, Smet KAG. 2015. RGB kleurmatching met nauwbandige en breedbandige primairen. KU Leuven, Campus Gent.

Friendly M, Sigal M. 2018. Visualizing Tests for Equality of Covariance Matrices. Am Stat.:123. doi:10.1080/00031305.2018.1497537.

Guild J. 1931. THE COLORIMETRIC PROPERTIES OF THE SPECTRUM. Encycl Earth Sci Ser. 230:149-187. doi:10.1007/978-90-481-2639-2_144.

Haddad HJ, Jakstat HA, Arnetzl G, Borbely J, Vichi A, Dumfahrt H, Renault P, Corcodel N, Pohlen B, Marada G, et al. 2009. Does gender and experience influence shade matching quality? J Dent. 37(SUPPL. 1):40-44. doi:10.1016/j.jdent.2009.05.012.

Henley SA, Fairchild MD. 2000. Quantifying mixed adaptation in cross-media color reproduction. In: Color and imaging conference. Vol. 2000. Society for Imaging Science and Technology. p. 305-310.

Houser KW, Hu X. 2004. Visually Matching Daylight Fluorescent Lamplight with Two Primary Sets†. Color Res Appl. 29(6):428-437. doi:10.1002/col.20059. 
Hu Y, Wei M, Luo MR. 2020. Observer metamerism to display white point using different primary sets. Opt Express. 28(14):20305. doi:10.1364/oe.395568.

Jain N, Verma P, Mittal S, Mittal S, Singh AK, Munjal S. 2010. Gender based alteration in color perception. Indian J Physiol Pharmacol. 54(4):366-370.

Judd DB. 1961. A five-attribute system of describing visual appearance. American Society for Testing Materials.

Katoh N, Nakabayashi K, Ito M, Ohno S. 1998. Effect of ambient light on color appearance of softcopy images: mixed chromatic adaptation for self-luminous displays. J Electron Imaging. 7(4):794-807.

Kuriki I. 2015. Effect of material perception on mode of color appearance. J Vis. 15(8):4. doi:10.1167/15.8.4. https://doi.org/10.1167/15.8.4.

Li J, Hanselaer P, Smet K. 2019a. THE IMPACT OF MATCHING PRIMARY PEAK WAVELENGTH ON COLOR MATCHING ACCURACY AND OBSERVER VARIABILITY. In: 27th Color and Imaging Conference Final Program and Proceedings, pp. 220-224 (5). Society for Imaging Science and Technology. p. 220-224.

Li J, Hanselaer P, Smet K. 2019b. PILOT STUDY ON COLOR MATCHING ACCURACY USING DIFFERENT PRIMARIES. In: Proceedings of the 29th CIE SESSION. p. 1010-1018.

Li J, Hanselaer P, Smet KAG. 2020. IMPACT OF COLOR MATCHING PRIMARIES ON OBSERVER MATCHING: PART I - ACCURACY. Submitted.

Long DL, Fairchild MD. 2014. Modeling Observer Variability and Metamerism Failure in Electronic Color Displays. J Imaging Sci Technol. 58(3):304021-3040214. doi:10.2352/J.ImagingSci.Technol.2014.58.3.030402.

http://openurl.ingenta.com/content/xref?genre=article\&issn=1062-

$3701 \&$ volume $=58 \&$ issue $=3 \&$ spage $=304021$.

Ma S, Hanselaer P, Teunissen K, Smet K. 2018. THE INFLUENCE OF ADAPTING FIELD SIZE ON DEGREE OF CHROMATIC ADAPTATION. In: CIE 2018 Smart Lighting, Date: 2018/04/24-2018/04/28, Location: Taipei.

Ma S, Hanselaer P, Teunissen K, Smet KAG. 2019. Impact of the starting point chromaticity on memory color matching accuracy. Opt Express. 27(24):35308. doi:10.1364/oe.27.035308.

Murdoch MJ, Fairchild MD. 2019. Modelling the effects of inter-observer variation on colour rendition. Light Res Technol. 51(1):37-54. doi:10.1177/1477153517744387.

Nayatani Y, Hashimoto K, Takahama K, Sobagaki H. 1985. Comparison of methods for assessing observer metamerism. Color Res Appl. 10(3):147-155.

Nimeroff I, Rosenblatt JR, Dannemiller MC. 1961. Variability of Spectral Tristimulus Values. J Res Natl Bur Stand Phys Chem. 65A(6):475-483. http://www.osapublishing.org/abstract.cfm?URI=josa-52-6-685.

North AD, Fairchild MD. 1993. Measuring color-matching functions. Part II. New data for assessing observer metamerism. Color Res Appl. 18(3):163-170. 
doi:10.1002/col.5080180306.

Prota G, Hu DN, Vincensi MR, McCormick SA, Napolitano A. 1998. Characterization of melanins in human irides and cultured uveal melanocytes from eyes of different colors. Exp Eye Res. 67(3):293-299. doi:10.1006/exer.1998.0518.

Rich DC, Jalijali J. 1995. Effects of Observer Metamerism in the Determination of Human Color-Matching Functions. Color Res Appl. 20(1):29-35. doi:10.1002/col.5080200106.

Sarkar A. 2011a. Final Report for R1-54: Variability In Color-Matching Functions.

Sarkar A. 2011b. Identification and Assignment of Colorimetric Observer Categories and Their Applications in Color and Vision Sciences. Université de Nantes. https://tel.archivesouvertes.fr/tel-00647246.

Sarkar A, Autrusseau F, Viénot F, Le Callet P, Blondé L. 2011. From CIE 2006 physiological model to improved age-dependent and average colorimetric observers. J Opt Soc Am A. 28(10):2033. doi:10.1364/josaa.28.002033.

Sarkar A, Blondé L, Le Callet P, Autrusseau F, Morvan P, Stauder J. 2010. Toward Reducing Observer Metamerism in Industrial Applications: Colorimetric Observer Categories and Observer Classification. In: Final Program and Proceedings - IS and T/SID Color Imaging Conference. p. 307-313.

Sarkar A, Blondé L, Le Callet P, Autrusseau F, Morvan P, Stauder J. 2010. A color matching experiment using two displays: Design considerations and pilot test results. 5th Eur Conf Colour Graph Imaging, Vis 12th Int Symp Multispectral Colour Sci 2010, CGIV 2010/MCS'10.:414-422.

Sarkar A, Blonde L, Le Callet P, Autrusseau F, Stauder J, Morvan P. 2009. Study of observer variability in modern display colorimetry: an analysis of CIE 2006 model. 11th Congr Int Colour Assoc.

Sarkar A, Blondé L, Le Callet P, Autrusseau F, Stauder J, Morvan P. 2009. Study of Observer Variability on Modern Display Colorimetry: Comparison of CIE 2006 Model and $10^{\circ}$ Standard Observer. In: The 11th Congress of the International Colour Association (AIC) . Sydney, Australia: AIC.

Shapiro AG, Pokorny J, Smith VC. 1994. Rod contribution to large-field color matching. Color Res Appl. 19(4):236-245. doi:10.1002/col.5080190404.

Shaw M, Fairchild M. 2002. Evaluating the 1931 CIE Color-Matching Functions. Color Res Appl. 27(5):316-329. doi:10.1002/col.10077.

Shi C, Huang M, Liu Y, Li Z, Liu H. 2017. Study on Observers' Categories Based on Color Matching Experiments. 417:123-129. doi:10.1007/978-981-10-3530-2. http://link.springer.com/10.1007/978-981-10-3530-2.

Smet KAG, Deconinck G, Hanselaer P. 2014. Chromaticity of unique white in object mode. Opt Express. 22(21):25830-25841.

Speranskaya NI. 1959. Determination of spectrum color coordinates for twenty-seven normal 
observers. Opt $\quad$ Spectrosc. 7:424-428. [accessed 2020 Mar 6]. http://ci.nii.ac.jp/naid/10018424674/en/.

Stiles WS, Burch JM. 1955a. Interim Report to the Commission Internationale de l'Eclairage, Zurich, 1955, on the National Physical Laboratory's Investigation of Colour-matching (1955). Opt Acta (Lond). 2(4):168-181. doi:10.1080/713821039.

Stiles WS, Burch JM. 1955b. Interim Report to the Commission Internationale de l'Eclairage, Zurich, 1955, on the National Physical Laboratory's Investigation of Colour- matching (1955). Opt Acta (Lond). 2(4):168-181. doi:10.1080/713821039.

Stiles WS, Burch JM. 1959. N.P.L. Colour-matching investigation: Final report (1958). Opt Acta Int J Opt. 6(1):1-26. doi:10.1080/713826267.

Stockman A, Sharpe LT. 2000. The spectral sensitivities of the middle- and long-wavelengthsensitive cones derived from measurements in observers of known genotype. Vision Res. 40(13):1711-1737. doi:10.1016/S0042-6989(00)00021-3.

Takahama K, Sobagaki H, Nayatani Y. 1985. Prediction of observer variation in estimating colorimetric values. Color Res Appl. 10(2):106-117.

Thornton WA. 1992. Toward a More Accurate and Extensible Colorimetry. Part I. Introduction. The Visual Colorimeter-Spectroradiometer. Experimental Results. Color Res Appl. 17(2):79-122. doi:10.1002/col.5080170208.

Thornton WA. 1997. Toward a More Accurate and Extensible Colorimetry. Part IV. Visual Experiments With Bright Fields and Both $10^{\circ}$ and $1.3^{\circ}$ Field Sizes William. Color Res Appl. 22(3):189-198. doi:10.1002/(SICI)1520-6378(199706)22:3<189::AID-COL7>3.0.CO;2-N.

Wielgus AR, Sarna T. 2005. Melanin in human irides of different color and age of donors. Pigment Cell Res. 18(6):454-464. doi:10.1111/j.1600-0749.2005.00268.x.

Wright WD. 1929. A re-determination of the trichromatic coefficients of the spectral colours. Trans Opt Soc. 30(4):141-164. doi:10.1088/1475-4878/30/4/301.

Wyszecki G ter., Stiles WS. 1982. Color science: concepts and methods, quantitative data and formulae. 2nd ed. John Wiley \& Sons. 